\title{
ON DISJOINT SETS OF DISTRIBUTION FUNCTIONS
}

\section{AGNES BERGER}

1. Introduction. Let us call a function $F(x)$ of the real variable $x$ a distribution function, $d . f$., if

$$
F(x) \text { is non-decreasing, continuous to the right, }
$$

$$
F(-\infty)=0, \quad F(+\infty)=1 \text {. }
$$

Let further $\left\{F_{i}(x)\right\}$ and $\left\{G_{j}(x)\right\}$ be two sets of d. f.'s with $i$ ranging over the set of values $I=\{i\}$ and $j$ over $J=\{j\}$. We shall assume

$$
\begin{aligned}
& F_{i}(x) \not \equiv F_{j}(x), \quad G_{i}(x) \not \equiv G_{j}(x), \quad \text { for all } i \text { and } j, \\
& F_{i}(x) \not \equiv G_{j}(x)
\end{aligned}
$$

and attempt to answer the following question proposed by Neyman:

What conditions must be satisfied by sets $\left\{F_{i}(x)\right\}$ and $\left\{G_{j}(x)\right\}$ so that there exists at least one Borel set $W$ such that

$$
\int_{W} d F_{i}(x) \neq \int_{W} d G_{j}(x) \quad \text { for all } i, j \text { ? }
$$

This question is of interest in the theory of statistical hypotheses. To indicate this briefly: Let $\xi$ be a random variable of which it is known only that its distribution belongs to the union of the two sets $\left\{F_{i}(x)\right\}$ and $\left\{G_{j}(x)\right\}$. Let $H$ be the hypothesis asserting that the distribution of $\xi$ belongs to $\left\{F_{i}(x)\right\}$. Should there be no Borel set $W$ of the property (A) it would cause difficulty in testing the hypothesis $\mathrm{H}$.

2. The simplest cases. To begin with we examine the simplest cases $I=1, J=1$ and $I=1, J=\{1,2\}$. It is easy to see that in these cases one can always find an interval solution for (A):

$I=1, J=1$. Since by $\left(C_{2}\right)$, there exists an $x_{0}$ such that $F_{1}\left(x_{0}\right)$ $\neq G_{1}\left(x_{0}\right)$, but by $\left(\mathrm{C}_{1}\right), F_{1}(-\infty)=G_{1}(-\infty)$, the interval $\left(-\infty, x_{0}\right)$ satisfies (A).

$I=1, J=\{1,2\}$. By $\left(C_{2}\right)$, either of the inequalities $F_{1}(x) \neq G_{j}(x)$, $j=1,2$, has at least one solution. If they have a simultaneous one, say $x_{0}$, then $\left(-\infty, x_{0}\right)$ satisfies (A), or else, if whenever $F_{1}\left(x_{1}\right) \neq G_{1}\left(x_{1}\right)$, we have $F_{1}\left(x_{1}\right)=G_{2}\left(x_{1}\right)$ and whenever $F_{1}\left(x_{2}\right) \neq G_{2}\left(x_{2}\right)$, then $F_{1}\left(x_{2}\right)$ $=G_{1}\left(x_{2}\right)$ so that $\left(x_{1}, x_{2}\right)$ satisfies (A).

Received by the editors August 23, 1948 and, in revised form, October 27, 1948. 
3. Discontinuous d. f.'s. So far no restrictions have been imposed upon the d.f.'s. Now we show that as long as the d. f.'s are allowed to have jumps, (A) does not always admit a solution when $I=1, J$ $=\{1,2,3\}$. Namely we shall prove the following theorem:

THeOREM I. Whatever be the distribution function $F(x)$, as long as it has at least two discontinuities, it is always possible, and in a continuum of ways, to determine three different distribution functions $G_{j}, j=1,2,3$, each different from $F(x)$ and such that for every Borel set $W$

$$
\int_{W} d F=\int_{W} d G_{j}
$$

for some $j$.

PRoof. Let $a_{1}<a_{2}$ be two discontinuity points of $F(x)$ and $a_{3}>a_{2}$ an arbitrary point on the $x$-axis. Let the set of three points $a_{1}, a_{2}, a_{3}$ be denoted by $\sigma$. Let the saltus of $F(x)$ at $a_{1}, a_{2}, a_{3}$ be respectively $p_{1}, p_{2}, p_{3}$, where $p_{1}>0, p_{2}>0$, but $p_{3} \geqq 0$ and $\sum_{i=1}^{3} p_{i}=s \leqq 1$. Then every Borel set $W$ can be represented as a sum $W^{\prime}+W^{\prime \prime}$, where $W^{\prime}=\sigma W$ and $W^{\prime \prime}=W-W^{\prime}$ and

$$
\int_{W} d F=\int_{W^{\prime}} d F+\int_{W^{\prime \prime}} d F \text {. }
$$

Let $a_{0}=-\infty, a_{4}=+\infty$. Let $G_{j}(x), j=1,2,3$, be three d. f.'s satisfying the relations $G_{j}(x)-G_{j}\left(a_{k}\right)=F(x)-F\left(a_{k}\right)$ for $a_{k} \leqq x<a_{k+1}$ for $k$ $=0,1,2,3$. Then, for every $W^{\prime \prime}$ disjoint with $\sigma$

$$
\int_{W^{\prime \prime}} d G_{j}(x)=\int_{W^{\prime \prime}} d F(x) \quad \text { for every } j .
$$

In order to complete the definition of the functions $G_{j}(x)$ it remains to specify their saltus at the points $a_{1}, a_{2}, a_{3}$. Thus let us denote the saltus of $G_{j}$ at $a_{i}$ by $q_{j i}, j, i=1,2,3$, and put

$$
q_{i j}=p_{j}, \quad j=1,2,3,
$$

while we choose arbitrarily any $q_{j i}$ such that

$$
0 \leqq q_{j i} \neq p_{i} \quad \text { for } i \neq j ; i, j=1,2,3,
$$

and satisfying $\sum_{i=1}^{3} q_{j i}=s, j=1,2,3$. Then $G_{j} \not \equiv F, j=1,2,3$, but for $W^{\prime}=a_{i}, i=1,2,3$,

$$
\int_{W^{\prime}} d F=p_{i}=\int_{W^{\prime}} d G_{i} \quad \text { for some } j .
$$

For $W^{\prime}=\sigma-a_{i}, i=1,2,3$, 


$$
\int_{W^{\prime}} d F=s-p_{j}=\int_{W^{\prime}} d G_{j} \quad \text { for some } j
$$

and since

$$
\int_{\sigma} d F=\int_{\sigma} d G_{j}
$$

for all $j$,

we have by $(*)$, for arbitrary $W$,

$$
\int_{W} d F=\int_{W} d G_{j} \quad \text { for some } j .
$$

Using Theorem $I$, we can show now that also in the case $I=\{1,2\}$, $J=\{1,2\}$, there exist sets of discontinuous d. f.'s for which (A) can not be satisfied. Namely let $F, G_{1}, G_{2}, G_{3}$ be a set of d. f.'s satisfying Theorem I and suppose further that $p_{2}<q_{32}<2 p_{2}$. (This is obviously allowed by the construction.) Then $p_{i}+q_{2 i}-q_{3 i} \geqq 0$ for $i=1,2,3$, so that the function $F(x)+G_{2}(x)-G_{3}(x)$ is a d. $\mathrm{f}$.

Let now

$$
F_{1}^{*}=F, \quad F_{2}^{*}=F+G_{2}-G_{3}, \quad G_{1}^{*}=G_{1}, \quad G_{2}^{*}=G_{2} .
$$

The sets $\left\{F_{i}^{*}\right\},\left\{G_{j}^{*}\right\}, i=1,2, j=1,2$, obviously satisfy $\left(C_{1}\right)$ and $\left(\mathrm{C}_{2}\right)$. Let $F-G_{j}=D_{j}, j=1,2,3$, and $F_{i}^{*}-G_{j}^{*}=D_{u}^{*}, i=1,2, j=1,2$. Then

$$
D_{11}^{*}=D_{1}, \quad D_{12}^{*}=D_{2}, \quad D_{21}^{*}=F+G_{2}-G_{3}-G_{1}, \quad D_{22}^{*}=D_{3} \text {, }
$$

so that the set $\left\{D_{v}^{*}\right\}, i=1,2, j=1,2$, contains the set $\left\{D_{j}\right\}, j=1,2,3$. Since, by assumption, whatever be $W, \int_{W} d F-\int_{W} d G_{j}=\int_{W} d\left(F-G_{j}\right)$ $=\int_{W} d D_{j}=0$ for at least one of the values $j=1,2,3$; also,

$$
\int_{W} d D_{i j}^{*}=0 \text { for at least one pair } i, j .
$$

Therefore we can state the following theorem:

THEOREM II. Whenever the number of distribution functions in the set $\left\{F_{i}\right\}$ multiplied by the number of distribution functions in the set $\left\{G_{j}\right\}$ is not less than 3 and the distribution functions are allowed to have jumps, it is not true that to every such pair of disjoint sets of distribution functions a Borel set $W$ exists for which

$$
\int_{W} d F_{i}-\int_{W} d G_{j} \neq 0 \quad \text { for all } i, j .
$$


4. Continuous distribution functions. On the other hand we shall show that if the d. f.'s are continuous, the following theorem holds:

TheOREM III. Let $\left\{F_{i}\right\}$ and $\left\{G_{j}\right\}$ be two denumerable sequences of distribution functions, each satisfying $\left(\mathrm{C}_{1}\right)$ and $\left(\mathrm{C}_{2}\right)$ and each continuous in $x$. Then there always exists a Borel set $W$ of arbitrarily small measure and such that

$$
\int_{W} d F_{i}-\int_{W} d G_{j} \neq 0 \quad \text { for all } i, j .
$$

In the proof we shall make repeated use of the terms "point of change of a function" and "interval of change of a function," defined as follows:

Let $f$ be any function defined on a closed interval $[X, Y]$ and such that

$$
f(X) \neq f(Y) .
$$

Let $Z$ be the least upper bound of those points $z$ of the interval $[X, Y]$ for which $f(z) \neq f(Y) . Z$ will be called the point of change of $f$ in $[X, Y]$. Obviously $Z$ has the property that given any $\delta>0$, there exists a point $z$ for which

$$
|z-Z|<\delta
$$

and

$$
f(z) \neq f(Z) .
$$

We shall say that $z$ and $Z$ determine an interval of change of $f$ in the $\delta$-neighborhood of $Z$.

Proof of Theorem III. Let $F_{i}(x)-G_{j}(x)=D_{i j}(x)$. Since

$$
\int_{W} d F_{i}-\int_{W} d G_{i}=\int_{W} d\left(F_{i}-G_{j}\right),
$$

we have to find a solution for

$$
\int_{W} d D_{i j}(x) \neq 0 \quad \text { for all } i, j .
$$

Since the sequences $\left\{F_{i}\right\}$ and $\left\{G_{j}\right\}$ are enumerable, so is the set $\left\{D_{i j}\right\}$ and its elements may be enumerated in a single indexed sequence

$$
D_{1}(x), D_{2}(x), \cdots, D_{i}(x), \cdots
$$


Thus $\left(A^{\prime}\right)$ becomes

$$
\int_{W} d D_{i}(x) \neq 0
$$

for all $i$

or, introducing the notation

$$
\begin{gathered}
\int_{W} d D_{i}(x)=V_{i}(W), \\
V_{i}(W) \neq 0
\end{gathered}
$$

for all $i$.

By $\left(C_{1}\right),\left(C_{2}\right)$, the functions $D_{i}(x)$ satisfy the following conditions:

$$
\begin{aligned}
& D_{i}(-\infty)=D_{i}(+\infty)=0, \\
& D_{i}\left(X_{i}\right) \neq 0 \quad \text { for at least one finite point } X_{i} .
\end{aligned}
$$

But since $D_{i}(x)$ is continuous, for each $D_{i}(x)$ there exists at least one other finite point $Y_{i}$ such that $D_{i}\left(Y_{i}\right) \neq 0$ and

$$
D_{i}\left(X_{i}\right) \neq D_{i}\left(Y_{i}\right) \text {. }
$$

Therefore we can assign to every $D_{i}(x)$ a point of change $Z_{i}$ and to every given $\delta$ an interval of change in the $\delta$-neighborhood of $Z_{i}$.

Let now $\epsilon>0$ be given in advance. First we shall show that it is possible to construct a sequence of Borel sets $W_{1}, W_{2}, \cdots, W_{r}, \cdots$ with the following properties:

(i) $W_{r}$ consists of a finite number of disjoint intervals;

(ii) The measure of $W_{r}$ is smaller than $\epsilon / 2^{r}$;

(iii) $V_{1}\left(W_{1}\right) \neq 0$;

(iv) If $V_{i}\left(W_{1}\right) \neq 0$ for $i \leqq i_{1}$ but $V_{i_{1}+1}\left(W_{1}\right)=0$, then there exists an $i_{2}>i_{1}$ such that $V_{i}\left(W_{2}\right) \neq 0$ for $i \leqq i_{2}$ and, in general, if $V_{i}\left(W_{r}\right) \neq 0$ for $i \leqq i_{r}$, but $V_{i_{r}+1}\left(W_{r}\right)=0$, then $V_{i}\left(W_{r+1}\right) \neq 0$ for $i \leqq i_{r+1}>i_{r}$.

If in the process of construction we arrive at a $W_{r}$ such that $V_{i}\left(W_{r}\right) \neq 0$ for all $i$, the theorem is proved. If, however, we arrive at an infinite sequence, we shall show that this sequence has a nonempty limit set $W$ which satisfies the requirements of the theorem.

The construction of the sequence $W_{r}$ is done by induction: Let $Z_{1}$ be a point of change of $D_{1}$ and $W_{1}$ an interval of change of $D_{1}$ in the $\epsilon / 2$-neighborhood of $Z_{1}$. Then (iii) holds. Assume now that we succeeded in finding $r$ terms of the sequence, $W_{1}, W_{2}, \cdots, W_{\text {, }}$, $W_{\text {s+1 }}, \cdots, W_{\mathrm{r}}$ such as requested. Then we know that

$$
\begin{aligned}
& V_{i}\left(W_{s}\right) \neq 0, \quad i \leqq i_{s}, \quad \text { for } s=1,2, \cdots, r, \\
& V_{i_{s}+1}\left(W_{s}\right)=0 .
\end{aligned}
$$

Let us for brevity put $i_{r}+1=\nu$ and let $Z$, be a point of change of $D_{\nu}$. 
Since by assumption $W_{r}$ consists of a finite number of disjoint intervals, we can and should choose a $\delta_{r}$ such that $0<\delta_{r}<\epsilon / 2^{r}$ and so small that the $\delta_{r}$-neighborhood of $Z_{v}$, and therefore every interval of change in it, should not contain any boundary point of $W_{r}$ except perhaps $Z_{\boldsymbol{y}}$.

By (1) for every $s \leqq r$, there exists an $\eta_{\bullet}>0$ such that

$$
\left|V_{i}\left(W_{s}\right)\right|>\eta_{s}, \quad i \leqq i_{s}, s \leqq r .
$$

Let now $\sum_{1}^{\infty} \alpha_{i}=\alpha<1, \alpha_{i}>\alpha_{i+1}>0$, be any given series and $\left[A_{v}, B_{v}\right]$ any closed interval $A_{v}<Z_{\nu}<B_{\nu}$. Since $D_{1}, D_{2}, \cdots, D_{i_{r}}$ are continuous in $\left[A_{v}, B_{v}\right], \delta_{r}$ can and should be further adjusted to be so small, that for every subinterval $I\left(\delta_{r}\right)$ of length smaller than $\delta_{r}$ in $\left[A_{v}, B_{v}\right]$

$$
\left|V_{i}\left[I\left(\delta_{r}\right)\right]\right|<\eta_{\ell} \alpha_{r}, \quad s \leqq r, i \leqq i_{r},
$$

should hold.

Having $\delta_{r}$ thus fixed, let $\Delta_{r}$ be an interval of change of $D_{v}$ in the $\delta_{r}$-neighborhood of $Z_{\eta}$, so that

$$
\left|\Delta_{r}\right|<\delta_{r}<\epsilon / 2^{r}
$$

and

$$
\left|V_{r}\left(\Delta_{r}\right)\right|=\left|V_{i_{r}+1}\left(\Delta_{r}\right)\right|>0
$$

Let now

$$
W_{r+1}=W_{r}+(-1)^{p_{r}} \Delta_{r},
$$

where $p_{r}=2$ if $\Delta_{r}$ contains no inner points of $W_{r}$, and $p_{r}=1$ if $\Delta_{r}$ contains inner but no outer points of $W_{r}$. Then by (3), (4), and (5)

$$
\left|V_{i}\left(W_{r+1}\right)\right| \geqq\left|V_{i}\left(W_{r}\right)\right|-\left|V_{i}\left(\Delta_{r}\right)\right|>\eta_{r}-\eta_{r} \alpha_{r}>0 \quad \text { for } i \leqq i_{r},
$$

and by (2) and (6)

$$
\left|V_{i_{r}+1}\left(W_{r+1}\right)\right|=\left|V_{i_{r}+1}\left(\Delta_{r}\right)\right|>0 .
$$

Let us now put

$$
W=W_{1}+\sum_{1}^{\infty}(-1)^{p_{r} \Delta_{r}}
$$

By (5), $W$ is not empty and its measure is less than $\epsilon$. By (4) we have (7) $\left|V_{i}\left(\Delta_{r}\right)\right|<\left|V_{i}\left(W_{s}\right)\right| \alpha_{r}, i \leqq i_{s}, s=1,2, \ldots, r ; r=1,2, \cdots$.

Let $i$ be a fixed arbitrary index. Find in the sequence $i_{1}, i_{2}, \cdots$ the indices $i_{k-1}, i_{k}$ for which 


$$
i_{k-1}<i \leqq i_{k}
$$

By (7)

$$
\left|V_{i}(W)\right| \geqq\left|V_{i}\left(W_{k}\right)\right|-\sum_{j=0}^{\infty}\left|V_{i}\left(\Delta_{k+j}\right)\right|,
$$

while for any $j$

$$
\left|V_{i}\left(\Delta_{k+j}\right)\right|<\left|V_{i}\left(W_{k}\right)\right| \alpha_{k+j}
$$

thus

$$
\sum_{j=0}^{\infty}\left|V_{i}\left(\Delta_{k+j}\right)\right|<\left|V_{i}\left(W_{k}\right)\right| \sum_{0}^{\infty} \alpha_{k+j}
$$

and

$$
\begin{gathered}
\left|V_{i}(W)\right|>\left|V_{i}\left(W_{k}\right)\right|\left(1-\sum_{0}^{\infty} \alpha_{k+j}\right) \geqq 0, \\
\left|V_{i}(W)\right|>0
\end{gathered}
$$

Q.E.D.

Further results concerning the case when $I$ and $J$ range over a continuum of indices are discussed in a joint paper with Wald in one of the recent issues of the Annals of Mathematical Statistics.

While preparing this paper I had opportunities to discuss it with Professor Neyman and I wish to acknowledge my indebtedness for his generous advice and many helpful suggestions.

New York City 